\title{
On a New Modification of Homotopy Analysis Method for Solving Nonlinear Nonhomogeneous Differential Equations
}

\author{
Shaheed N. Huseen ${ }^{1}$ and Haider A. Mkharrib ${ }^{2}$ \\ University of Thi-Qar, Faculty of Computer Science and Mathematics, \\ Mathematics Department, Thi-Qar, Iraq \\ shn_n2002@yahoo.com ${ }^{1}$ \\ haider.4ali91@gmail.com²
}

\begin{abstract}
In this paper, new powerful modification of homotopy analysis technique (NMHAM) was submitted to create an approximate solution of nonhomogeneous nonlinear ordinary and partial differential equations. The NMHAM is a combination of the new technique of homotopy analysis method(NHAM) [4] and the new technique of homotopy analysis method(nHAM) [7]. Three illustrative examples are employed to illustrate the accuracy and computational proficiency of this approach. The outcomes uncover that the NMHAM is more accurate than the NHAM and nHAM.
\end{abstract}

Keywords: Modified homotopy analysis method, Taylor Series, Nonhomogeneous differential equations.

\section{Introduction}

In recent years, many engineers and scientists in various sciences like Mathematics, Biology,Physics, and particularly in branches of engineering like Fluid mechanics, Numerical calculations in Aerospace and Electronics are faced with nonlinear phenomena and many nonlinear problems. Since solving nonlinear problems plays a crucial role in various fields of engineering and science, Scientists are interested in obtaining techniques for solving nonlinear problems and have performed extensive researchers to achieve nonlinear problem solving techniques. As solving nonlinear problems are generally difficult and achieving their exact solutions are hard, various approximate methods have been developed to solve them.

The homotopy analysis technique (HAM), proposed by Liao [14], is a powerful technique to solve non-linear problems. In recent years, this method has been effectively applied to numerous problems in science and engineering [15-27]. All of these successful applications verified the validity, effectiveness and flexibility of the HAM. Recently, some modifications of HAM have published to facilitate and accurate the calculations and accelerate the rapid convergence of the series solution and reduce the size of work [1-13]. It is the aim of this paper to submit a new powerful modification of the HAM. The NMHAM is a combination of the two modifications of homotopy analysis technique (NHAM) [4] and the nHAM [7]. The NMHAM demonstrates an accurate solution if compared with the NHAM and nHAM, and therefore it has been shown that to be computationally efficient in applied fields. The obtained results suggest that this newly improvement technique introduces a powerful improvement for solving nonlinear problems.

\section{The New Technique of Homotopy Analysis Method (NHAM)}

Consider the following nonlinear differential equation 
$\mathbb{N}[y(x, t)]=f(x, t)$

Where $\mathbb{N}$ is a nonlinear operator, $(x, t)$ means independent variables, $y(x, t)$ is an unknown function, and $f(x, t)$ is a non-homogeneous terms.

The non-homogeneous terms $f(x, t)$ in $(2.1)$ can be expressed in Taylor series based on a kind of continuous homotopy mapping with respect to $\mathbb{q}$, where $\mathbb{q} \in[0,1]$ is an embedding parameter, $f(x, t) \rightarrow \mu(x, t ; \mathbb{q})$ as [4]

$\mu(x, t ; \mathbb{q})=\sum_{r=0}^{\infty} f_{r}{ }^{s}(x, t) \mathbb{q}^{r}=f_{0}{ }^{s}(x, t) \mathbb{q}^{0}+f_{1}{ }^{s}(x, t) \mathbb{q}^{1}+\cdots+f_{n}{ }^{s}(x, t) \mathbb{q}^{n}+\cdots$

Where

$$
\begin{gathered}
f_{r}^{s}(x, t)=\frac{1}{r s !}\left[\frac{d^{r s}}{d t^{r s}} f(x, t)\right]_{t=0} t^{r s}+\frac{1}{(r s+1) !}\left[\frac{d^{(r s+1)}}{d t^{(r s+1)}} f(x, t)\right]_{t=0} t^{(r s+1)}+\cdots \\
+\frac{1}{(r s+s-1) !}\left[\frac{d^{(r s+s-1)}}{d t^{(r s+s-1)}} f(x, t)\right]_{t=0} t^{(r s+s-1)}
\end{gathered}
$$

We note that $f_{r}{ }^{s}(x, t)$ depend on the order of the differential equation s.For example,

$$
\begin{aligned}
s=1 \Rightarrow f_{r}{ }^{1} & =\frac{1}{r !}\left[\frac{d^{r}}{d t^{r}} f(x, t)\right]_{t=0} t^{r}, \\
s=2 \Rightarrow f_{r}{ }^{2} & =\frac{1}{2 r !}\left[\frac{d^{2 r}}{d t^{2 r}} f(x, t)\right]_{t=0} t^{2 r}+\frac{1}{(2 r+1) !}\left[\frac{d^{(2 r+1)}}{d t^{(2 r+1)}} f(x, t)\right]_{t=0} t^{(2 r+1)}, \\
s & =4 \Rightarrow f_{r}^{2}=\frac{1}{4 r !}\left[\frac{d^{4 r}}{d t^{4 r}} f(x, t)\right]_{t=0} t^{4 r}+\frac{1}{(4 r+1) !}\left[\frac{d^{(4 r+1)}}{d t^{(4 r+1)}} f(x, t)\right]_{t=0} t^{(4 r+1)} \\
& +\frac{1}{(4 r+2) !}\left[\frac{d^{(4 r+2)}}{d t^{(4 r+2)}} f(x, t)\right]_{t=0} t^{(4 r+2)}+\frac{1}{(4 r+3) !}\left[\frac{d^{(4 r+3)}}{d t^{(4 r+3)}} f(x, t)\right]_{t=0} t^{(4 r+3)}
\end{aligned}
$$

Give us a chance to develop the supposed zeroth deformation equation as follows

$$
(1-\mathbb{q}) \mathrm{L}\left[\delta(x, t ; \mathfrak{q})-y_{0}(x, t)\right]=q \mathbb{l} \mathbb{H} \mathcal{H}(x, t) \mathbb{N}[\delta(x, t ; \mathfrak{q})-\mu(x, t ; \mathfrak{q})]
$$

Where $\mathbb{q} \in[0,1]$ is an embedding parameter, $\mathbb{h} \neq 0$ is an auxiliary parameter, L is an auxiliary linear operator, $\mathrm{y}_{0}(\mathrm{x}, \mathrm{t})$ is the initial guesses of $y(x, t), \delta(\mathrm{x}, \mathrm{t} ; \mathrm{q})$ is an unknown functions, and $\mathcal{H}(x, t)$ denotes a nonzero auxiliary function. It is evident that when $\mathbb{q}=0$ and $\mathbb{q}=1$ becomes

$\delta(x, t ; 0)=y_{0}(x, t), \quad \delta(x, t ; 1)=y(x, t)$

respectively. In this way as $\mathbb{q}$ increments from 0 to 1 , the solution $\delta(x, t ; \mathbb{q})$ varies from the initial guess $y_{0}(x, t)$ to the solution $y(x, t)$. Having the freedom to select $y_{0}(x, t), \mathrm{L}$, $\mathbb{h}$, and $\mathcal{H}(x, t)$, we can expect that every one of them can be chosen with the goal that the solution $\delta(x, t ; \mathbb{q})$ of (2.4) exists for $\mathbb{q} \in[0,1]$.

Expanding $\delta(x, t ; q)$ in Taylor series, we have 
$\delta(x, t ; q)=y_{0}(x, t)+\sum_{r=1}^{+\infty} y_{r}(x, t) \mathbb{q}^{r}$,

Where

$y_{r}(x, t)=\left.\frac{1}{r !} \frac{\partial^{r} \delta(x, t ; q)}{\partial \mathbb{I}^{r}}\right|_{\mathbb{q}=0}$.

Next, recall that $\mathbb{l n}, \mathcal{H}(x, t), y_{0}(x, t)$, and $\mathrm{L}$ are select with the end goal that the series (2.6) converges at $\mathbb{q}=1$ and that

$y(x, t)=\delta(x, t ; 1)=y_{0}(x, t)+\sum_{r=1}^{+\infty} y_{r}(x, t)$

Let $y_{e}(x, t)=\left\{y_{0}(x, t), y_{1}(x, t), y_{2}(x, t), \ldots y_{e}(x, t)\right\}$.

Differentiating equation (2.4) for $r$ times with respect to $\mathbb{q}$ and afterward setting $\mathbb{q}=0$ and lastly dividing the resulting equation by $r$ ! , we have the so-called $r$ th order deformation equation as follows:

$L\left[y_{r}(x, t)-X_{r} y_{r-1}(x, t)\right]=\mathbb{h} \mathcal{H}(x, t) \mathcal{R}_{r}\left(\overrightarrow{y_{r-1}}(x, t)\right)$,

where

$\mathcal{R}_{r}\left(\overrightarrow{y_{r-1}}(x, t)\right)=\left.\frac{1}{(r-1) !} \frac{\partial^{r-1}(\mathbb{N}[\delta(x, t, \mathbb{q})]-\mu(x, t ; q))}{\partial \mathbb{q}^{r-1}}\right|_{\mathbb{q}=0}$

And $X_{r}= \begin{cases}0, & r \leq 1, \\ 1, & r>1 .\end{cases}$

It ought to be underscored that $y_{r}(x, t)$ for $r \geq 1$ is administered by the equation (2.10) with the boundary conditions that come from the original problem.

The homogenous part of equation (2.1) can be written as [7]

$$
\begin{gathered}
L y(x, t)+\operatorname{Ay}(x, t)+\operatorname{By}(x, t)=0, \\
y(x, 0)=g_{0}(x), \\
\left.\frac{\partial y(x, t)}{\partial t}\right|_{t=0}=g_{1}(x), \\
\vdots \\
\left.\frac{\partial^{k-1} y(x, t)}{\partial^{k-1}}\right|_{t=0}=g_{k-1}(x) .
\end{gathered}
$$

Where $\mathrm{L}=\partial^{k} / \partial t^{k}, k=1,2, \ldots$ is the highest partial derivative with respect to $\mathrm{t}, \mathrm{A}$ is a linear term, and B is a nonlinear term.

Hence, the equation (2.1) will be take the form: 


$$
\begin{gathered}
L y(x, t)+\operatorname{Ay}(x, t)+\operatorname{By}(x, t)=\mu(x, t ; \mathbb{q}), \\
y(x, 0)=g_{0}(x), \\
\left.\frac{\partial y(x, t)}{\partial t}\right|_{t=0}=g_{1}(x), \\
\vdots \\
\left.\frac{\partial^{k-1} y(x, t)}{\partial^{k-1}}\right|_{t=0}=g_{k-1}(x) .
\end{gathered}
$$

And the so-called zero-order deformation equation (2.4) becomes

$$
(1-\mathbb{q}) L\left[\delta(x, t ; q)-y_{0}(x, t)\right]=\mathbb{q} \mathbb{l} \mathcal{H}(x, t)(L y(x, t)+A y(x, t)+B y(x, t)-\mu(x, t ; q))
$$

Hence, the $r^{\text {th }}$ order deformation equation will becomes:

$$
\begin{aligned}
& L\left[y_{r}(x, t)-X_{r} y_{r-1}(x, t)\right]=\mathbb{h} \mathcal{H}(x, t)\left(L y_{r-1}(x, t)+A y_{r-1}(x, t)+B\left(\overrightarrow{y_{r-1}}(x, t)\right)-\right. \\
& \left.f_{r-1}^{s}(x, t)\right)
\end{aligned}
$$

Therefore

$$
\begin{aligned}
y_{r}(x, t) & =X_{r} y_{r-1}(x, t)+\mathbb{h} L^{-1}\left[\mathcal { H } ( x , t ) \left(L y_{r-1}(x, t)+A y_{r-1}(x, t)\right.\right. \\
& \left.\left.+B\left(y \overline{\lambda_{r-1}}(x, t)\right)-f_{r-1}{ }^{s}(x, t)\right)\right]
\end{aligned}
$$

Such that

$$
L^{-1}(.)=\iint \cdots \int(.) \underbrace{d t d t \cdots d t}_{\text {ktimes }}+c_{1} t^{k-1}+c_{2} t^{k-1}+\cdots+c_{k} .
$$

Where $c_{1}, c_{2}, \ldots, c_{k}$ are constants.

To solve (2.13) by means of HAM, we select the following initial approximation

$y_{0}(x, t)=g_{0}(x)+g_{1}(x) t+g_{2}(x) \frac{t^{2}}{2 !}+\cdots+g_{k-1}(x) \frac{t^{k-1}}{(k-1) !}$.

Let $\mathcal{H}(x, t)=1$, by means of (2.17) and (2.18); then (2.16) becomes

$$
\begin{aligned}
y_{r}(x, t)= & X_{r} y_{r-1}(x, t)+\mathbb{h} \int_{0}^{t} \int_{0}^{t} \ldots \int_{0}^{t}\left(\frac{\partial^{k} y_{r-1}(x, \tau)}{\partial \tau^{k}}+A y_{r-1}(x, \tau)+B\left(\overline{y_{r-1}}(x, \tau)\right)-\right. \\
& \left.f_{r-1}^{s}(x, \tau)\right) \underbrace{d \tau d \tau \ldots d \tau}_{\text {ktimes }} .
\end{aligned}
$$

Now we have 


$$
\begin{aligned}
y_{r}(x, t)= & X_{r} y_{r-1}(x, t)+\mathbb{h} \int_{0}^{t} \int_{0}^{t} \ldots \int_{0}^{t} \frac{\partial^{k} y_{r-1}(x, \tau)}{\partial \tau^{k}} \underbrace{d \tau d \tau \ldots d \tau}_{k t i m e s}+\mathbb{h} \int_{0}^{t} \int_{0}^{t} \ldots \int_{0}^{t} A y_{r-1}(x, \tau)+ \\
& \left.B\left(\overline{y_{r-1}}(x, \tau)\right)-f_{r-1}{ }^{s}(x, \tau)\right) \underbrace{d \tau d \tau \ldots d \tau}_{k t i m e s} \\
= & X_{r} y_{r-1}(x, t)+\mathbb{h} y_{r-1}(x, t)-\mathbb{h}\left(y_{r-1}(x, 0)+t \frac{\partial y_{r-1}(x, 0)}{\partial t}+\cdots+\frac{t^{k-1} \partial^{k-1} y_{r-1}(x, 0)}{(k-1) !}\right) \\
+ & \mathbb{h} \int_{0}^{t} \int_{0}^{t} \ldots \int_{0}^{t}\left(A y_{r-1}(x, \tau)+B\left(\overline{y_{r-1}}(x, \tau)\right)-f_{r-1}{ }^{s}(x, \tau)\right) \underbrace{d \tau d \tau \ldots d \tau}_{\text {ktimes }}
\end{aligned}
$$

For $r=1, X_{r}=0$, and

$$
\begin{aligned}
& y_{0}(x, 0)+t \frac{\partial y_{0}(x, 0)}{\partial t}+\frac{t^{2}}{2 !} \frac{\partial^{2} y_{0}(x, 0)}{\partial t^{2}}+\cdots+\frac{t^{k-1}}{(k-1) !} \frac{\partial^{k-1} y_{0}(x, 0)}{\partial t^{k-1}} \\
= & g_{0}(x)+g_{1}(x) t+g_{2}(x) \frac{t^{2}}{2 !}+\cdots+g_{k-1}(x) \frac{t^{k-1}}{(k-1) !} \\
= & y_{0}(x, t)
\end{aligned}
$$

Substituting this equality into (2.20), we obtain

$\left.y_{1}(x, t)=\mathbb{h} \int_{0}^{t} \int_{0}^{t} \ldots \int_{0}^{t} A y_{0}(x, \tau)+B\left(\overrightarrow{y_{0}}(x, \tau)\right)-f_{0}{ }^{s}(x, \tau)\right) \underbrace{d \tau d \tau \ldots d \tau}_{k \text { times }}$

For $r>1, X_{r}=1$, and

$y_{r}(x, 0)=0, \frac{\partial y_{r}(x, 0)}{\partial t}=0, \frac{\partial^{2} y_{r}(x, 0)}{\partial t^{2}}=0, \cdots, \frac{\partial^{(k-1)} y_{r}(x, 0)}{\partial t^{(k-1)}}=0$.

Substituting this equality into (2.20), we obtain

$$
\begin{gathered}
y_{r}(x, t)=(1+\mathbb{h}) y_{r-1}(x, t)+\mathbb{h} \int_{0}^{t} \int_{0}^{t} \ldots \int_{0}^{t}\left(A y_{r-1}(x, \tau)+B\left(\overline{y_{r-1}}(x, \tau)\right)-\right. \\
\left.f_{r-1}^{s}(x, \tau)\right) \underbrace{d \tau d \tau \ldots d \tau}_{\text {ktimes }}
\end{gathered}
$$

The NHAM is powerful when $k=1$, and the solution of NHAM can be written as the following series:

$y(x, t ; \mathbb{h})=Y_{r}(x, t ; \mathfrak{h})=\sum_{i=0}^{r} y_{i}(x, t ; \mathbb{h})$

But when $k \geq 2$, there are too many additional terms where harder and more timeconsuming computations are performed.so, the closed form solution needs more numbers of iteration.

\section{The New Modified Homotopy Analysis Method (NMHAM)}

When $k \geq 2$, we rewrite (2.1) as in the following system: 


$$
\begin{aligned}
y_{t} & =y 1 \\
y 1_{t} & =y 2 \\
& \vdots \\
y\{k-1\}_{t} & =-A y(x, t)-B y(x, t)+f(x, t)
\end{aligned}
$$

Set the initial approximation

$$
\begin{aligned}
y_{0}(x, t) & =g_{0}(x) \\
y 1_{0}(x, t) & =g_{1}(x) \\
& \vdots \\
y\{k-1\}_{0}(x, t) & =g_{k-1}(x)
\end{aligned}
$$

We note that the order of differential equation (3.1) is the first order $(s=1)$ Since all the equations of the system (3.1) of the first order then The non-homogeneous terms $f(x, t)$ in the last equation of (3.1) can be expressed in Taylor series based on a kind of continuous homotopy mapping with respect to $\mathbb{q}, f(x, t) \rightarrow \mu(x, t ; \mathbb{q})$ as the following

$\mu(x, t ; \mathbb{q})=\sum_{r=0}^{\infty} f_{r}{ }^{1}(x, t) \mathbb{q}^{r}=f_{0}{ }^{1}(x, t) \mathbb{q}^{0}+f_{1}{ }^{1}(x, t) \mathbb{q}^{1}+\cdots+f_{n}{ }^{1}(x, t) \mathbb{q}^{n}+\cdots$

Where

$f_{r}^{1}=\frac{1}{r !}\left[\frac{d^{r}}{d t^{r}} f(x, t)\right]_{t=0} t^{r}$

Using the iteration formulas (2.22) and (2.24) as follows:

$$
\begin{aligned}
y_{1}(x, t) & =\mathbb{h} \int_{0}^{t}\left(-y 1_{0}(x, \tau)\right) d \tau \\
y 1_{1}(x, t) & =\mathbb{h} \int_{0}^{t}\left(-y 2_{0}(x, \tau)\right) d \tau \\
& \vdots \\
y\{k-1\}_{1}(x, t) & =\mathbb{h} \int_{0}^{t}\left(A y_{0}(x, \tau)+B\left(y_{0}(x, \tau)\right)-f_{0}^{1}(x, \tau)\right) d \tau
\end{aligned}
$$

For $r>1, X_{r}=1$, and

$y_{r}(x, 0)=0, y 1_{r}(x, 0)=0, y 2_{r}(x, 0)=0, \cdots, y\{k-1\}_{r}(x, 0)=0$.

Substituting in (2.20), we obtain 


$$
\begin{gathered}
y_{r}(x, t)=(1+\mathbb{h}) y_{r-1}(x, t)+\mathbb{h} \int_{0}^{t}\left(-y 1_{r-1}(x, \tau)\right) d \tau, \\
y 1_{r}(x, t)=(1+\mathbb{h}) y 1_{r-1}(x, t)+\mathbb{h} \int_{0}^{t}\left(-y 2_{r-1}(x, \tau)\right) d \tau, \\
\vdots \\
y\{k-1\}_{r}(x, t)=(1+\mathbb{h}) y\{k-1\}_{r-1}(x, t)+\mathbb{h} \int_{0}^{t}\left(A y_{r-1}(x, \tau)+B\left(y_{r-1}(x, \tau)\right)\right. \\
\left.-f_{r-1}^{1}(x, \tau)\right) d \tau
\end{gathered}
$$

\section{Applications}

4.1 Example 1 Consider a Duffings equation [4]

$\frac{d^{2} y}{d t^{2}}+3 y-2 y^{3}=f(t)$

Equation (4.1) with the initial condition

$\mathrm{y}(0)=0, y^{\prime}(0)=1$,

and $f(t)=\cos (t) \sin (2 t)$ has the exact solution

$y(t)=\sin (t)$

The problem (4.1)-(4.2) solved by ( NHAM )[4].

In order to solve (4.1)-(4.2) by the proposed approach (NMHAM) we construct the following system:

$y_{t}(t)=v(t)$,

$v_{t}(t)=-3 y+2 y^{3}+\mu(t ; \mathfrak{q})$

with the following initial conditions :

$y_{0}(t)=0, v_{0}(t)=1$

We expand the homotopy $\mu(t ; \mathfrak{q})$ in powers of the parameter $\mathbb{q}$ with $\mathrm{s}=1$ :

$\mu(t ; \mathfrak{q})=\sum_{r=0}^{\infty} f_{r}{ }^{1}(t) \mathbb{q}^{r}=f_{0}{ }^{1}(t) \mathbb{q}^{0}+f_{1}{ }^{1}(t) \mathbb{q}^{1}+\cdots+f_{n}{ }^{1}(t) \mathbb{q}^{n}+\cdots$

Where

$f_{r}^{1}(t)=\frac{1}{r !}\left[\frac{d^{r}}{d t^{r}} f(t)\right]_{t=0} t^{r}$

such that

$$
f_{0}{ }^{1}(t)=0 \quad, f_{1}{ }^{1}(t)=2 t \quad, f_{2}{ }^{1}(t)=0 \quad, f_{3}{ }^{1}(t)=-\frac{7}{3} t^{3}, \ldots
$$

and the following linear operators: 


$$
\begin{aligned}
& L y(t)=\frac{\partial y(t)}{\partial t}, L v(t)=\frac{\partial v(t)}{\partial t} \\
& A y_{r-1}(t)=3 y_{r-1}(t)-f^{1}{ }_{r-1}(t) \\
& B y_{r-1}(t)=-2 \sum_{i=0}^{r-1} y_{r-1-i} \sum_{j=0}^{i} y_{j} y_{i-j}
\end{aligned}
$$

we obtain

$y_{1}(t)=\mathbb{n} \int_{0}^{t}\left(-v_{0}(\tau)\right) d \tau$

$v_{1}(t)=\mathbb{h} \int_{0}^{t}\left(3 y_{0}(\tau)-2 y_{0}{ }^{3}(\tau)-f_{0}{ }^{1}(\tau)\right) d \tau$

Now, for $r \geq 2$, we get

$y_{r}(t)=(1+\mathbb{h}) y_{r-1}(t)+\mathbb{h} \int_{0}^{t}\left(-v_{r-1}(\tau)\right) d \tau$,

$v_{r}(t)=(1+\mathbb{h}) v_{r-1}(t)+\mathbb{h} \int_{0}^{t}\left(A y_{r-1}(\tau)+B y_{r-1}(\tau)\right) d \tau$

And the following results are obtained:

$y_{1}(t)=-\mathbb{h} t$

$v_{1}(t)=0$

$y_{2}(t)=-\mathbb{l}(1+\mathbb{h}) t$

$v_{2}(t)=\mathbb{h}\left(-t^{2}-\frac{3 h t^{2}}{2}\right)$

$y_{3}(t)=-\mathbb{h}(1+\mathbb{h})^{2} t+\mathbb{h}\left(\frac{\mathfrak{h t}^{3}}{3}+\frac{\mathbb{h}^{2} t^{3}}{2}\right)$

$\vdots$

Then, the series solution of the NMHAM is:

$y(t, \mathbb{h}) \cong Y_{R}(t, \mathbb{h})=\sum_{i=0}^{R} y_{i}(t, \mathbb{h})$

Equation (4.12) is an approximation solutions for the problem (4.1)-(4.2) depending on the parameter $\mathbb{h}$. To determine the valid region of , the $\mathbb{h}$-curves given by the $7^{\text {th }}$-order NMHAM at different values of $t$ are drawn in figure (1). 


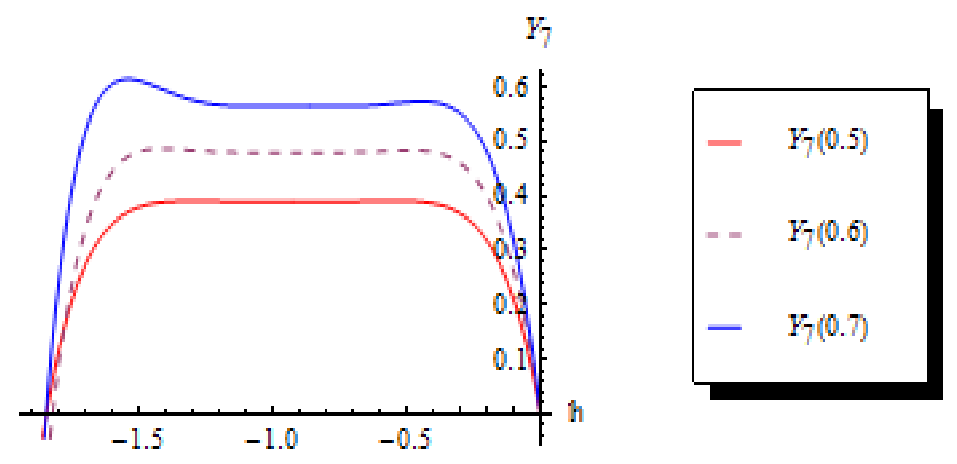

Figure(1) $\mathbb{h}$-curve for NMHAM approximation solutions $\mathrm{Y}_{7}(\mathrm{t})$ of problem (4.1) -(4.2)at different values of $\mathrm{t}$.

Figure(2) show the comparison between $Y_{7}$ of NMHAM and $Y_{7}$ of NHAM at $\leq t \leq 3.5$ with the exact solution(4.3) .Figure (3) comparison between $Y_{6}, Y_{4}$ of NMHAM and $Y_{7}$ of NHAM with the exact solution (4.3) at $0 \leq t \leq 3$ which indicates that the speed of convergence of NMHAM is faster in comparison of NHAM.

The absolute error of the $7^{\text {th }}$ order approximate solution of NMHAM compared with $7^{\text {th }}$ order approximate solution of NHAM are calculated by the formula

Absolute Error $(A . E)=\left|Y_{\text {exact }}-Y_{\text {approx }}\right|$

Figures (4) show that the series solution $Y_{7}$ obtained by NMHAM at $0 \leq t \leq 0.5$ is more accuratefrom the series solution $Y_{7}$ obtained byNHAM.Figures (5) show that the series solution $Y_{7}$ obtained by NMHAM is more accurate from the series solution $Y_{7}$ obtained by NHAM at larger $t$ $(0.5 \leq t \leq 1)$. Figures (6) show that the series solution $Y_{6}$ obtained by NMHAM at $0 \leq t \leq 0.5$ is moreand faster converge from the series solution $Y_{7}$ obtained by NHAM. Figures (7) show that the series solution $Y_{6}$ obtained by NMHAM is moreand faster converge from the series solution $Y_{7}$ obtained by NHAM at larger $t(0.5 \leq t \leq 1)$.

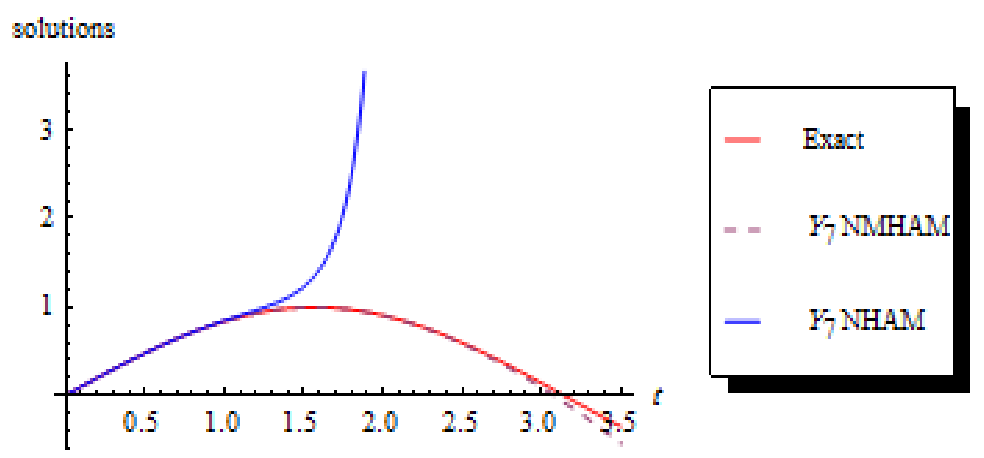

Figure(2): Comparison of the $7^{\text {th }}$ order approximations of NMHAM and NHAM at $0 \leq t \leq 3.5$, $\mathbb{h}=-1$ with the exact solution(4.3). 


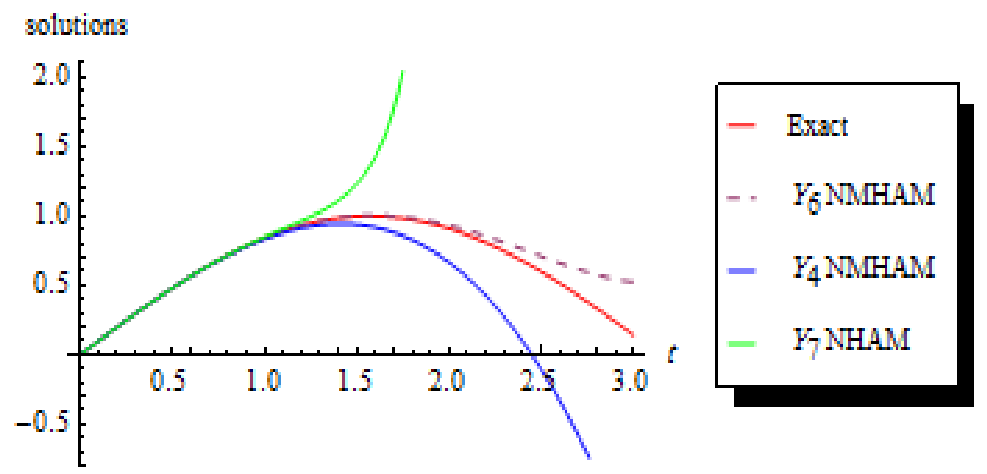

Figure(3):Comparison of $Y_{6}, Y_{4}$ of NMHAM and $Y_{7}$ of NHAM with the exact solution (4.3) at $0 \leq t \leq 3$, $\mathbb{h}=-1$.

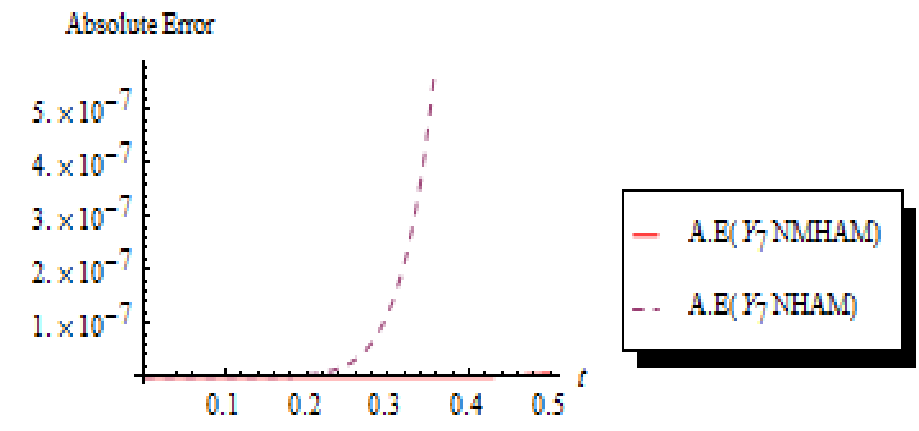

Figure(4):Absolute errors of $Y_{7}$ of NMHAM and $Y_{7}$ of NHAMat $0 \leq t \leq 0.5$, $\mathbb{h}=-1$.

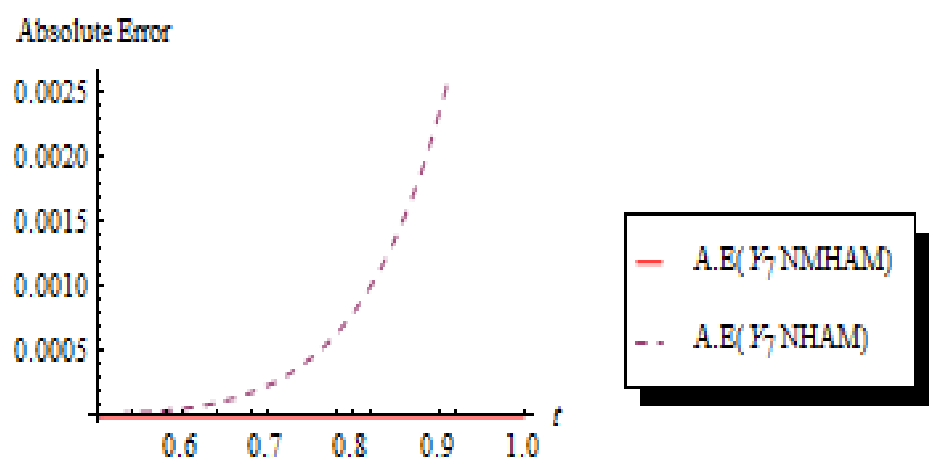

Figure(5):Absolute errors of $Y_{7}$ of NMHAM and $Y_{7}$ of NHAMat0.5 $\leq t \leq 1$, $\mathbb{h}=-1$. 


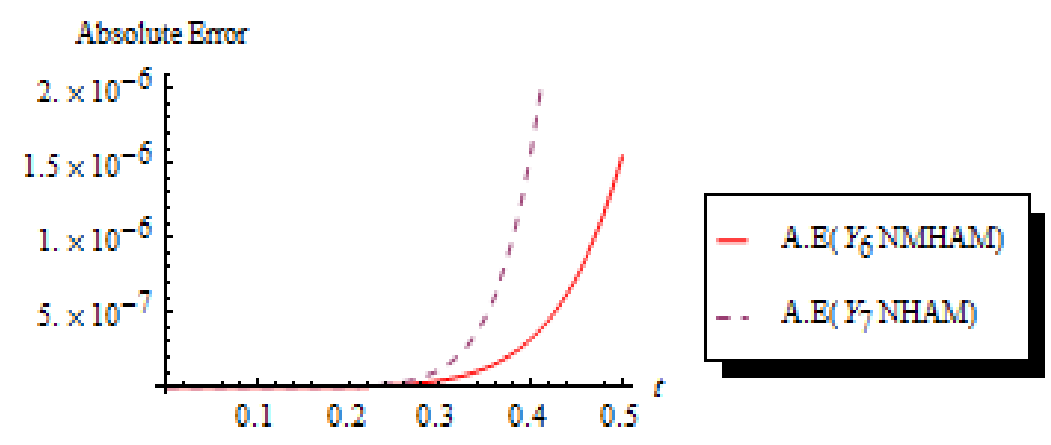

Figure(6):Absolute errors of $Y_{6}$ of NMHAM and $Y_{7}$ of NHAM at $0 \leq t \leq 0.5$, h $=-1$

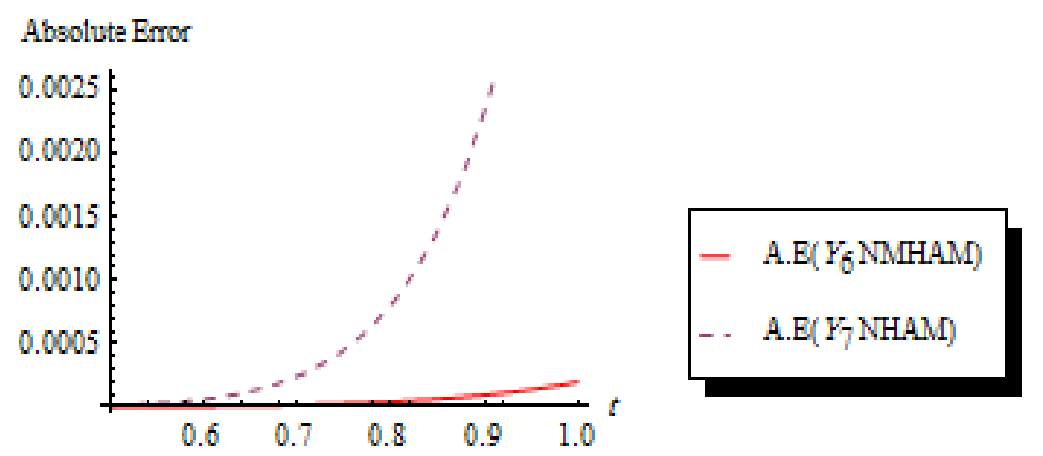

Figure(7):Absolute errors of $Y_{6}$ of NMHAM and $Y_{7}$ of NHAM at $0.5 \leq t \leq 1, \mathrm{hn}=-1$

Table (1) show the comparison of $Y_{7}$ of NMHAM ,NHAM and nHAM, with the exact solution(4.3) at different values of $t$.Table (2) show the comparison between the absolute errors of $Y_{7}$ of NMHAM ,NHAM and nHAM at different values of $t$. Tables (1) and (2) indicate that the series solution obtained by NMHAM is more accurate from the series solution obtained by NHAM and the series solution obtained by nHAMis divergent for all $t$ except $t=0$ and the absolute error monotonously increases very quickly.

Table1: Comparison of the $7^{\text {th }}$ order approximations of NMHAM $7^{\text {th }}$ order approximations of NHAM and $7^{\text {th }}$ order approximations of nHAM with the exact solution.

\begin{tabular}{|l|c|c|c|c|}
\hline $\boldsymbol{t}$ & exact & NMHAM & NHAM & nHAM \\
\hline 0 & 0 & 0 & 0 & 0 \\
\hline 0.2 & 0.198669 & 0.198669 & 0.198669 & -2.376868 \\
\hline 0.4 & 0.389418 & 0.389418 & 0.389419 & -4.709543 \\
\hline 0.6 & 0.564642 & 0.564642 & 0.564703 & -6.959303 \\
\hline 0.8 & 0.717356 & 0.717356 & 0.718162 & -9.10208 \\
\hline 1 & 0.841471 & 0.841468 & 0.847508 & -11.144744 \\
\hline 1.2 & 0.932039 & 0.932025 & 0.963396 & -13.152658 \\
\hline
\end{tabular}




\begin{tabular}{|l|l|l|l|l|}
\hline 1.4 & 0.985449 & 0.985393 & 1.111087 & -15.294508 \\
\hline 1.6 & 0.999574 & 0.999389 & 1.424605 & -17.912939 \\
\hline 1.8 & 0.973848 & 0.973317 & 2.459322 & -21.629384 \\
\hline 2 & 0.909297 & 0.907937 & 7.466350 & -27.489478 \\
\hline
\end{tabular}

Table2: Comparisonof the Absolute errors of $7^{\text {th }}$ order approximations of NMHAM , $7^{\text {th }}$ order approximations of NHAM and $7^{\text {th }}$ order approximations of nHAM with the exact solution

\begin{tabular}{|l|c|c|c|}
\hline $\boldsymbol{t}$ & $\boldsymbol{A . E}$ NMHAM & A.E NHAM & A.E $\boldsymbol{n H A M}$ \\
\hline 0 & 0 & 0 & 0 \\
\hline 0.2 & $1.410399 \times 10^{-12}$ & $3.083948 \times 10^{-9}$ & 2.575537 \\
\hline 0.4 & $7.213487 \times 10^{-10}$ & $1.575220 \times 10^{-6}$ & 5.098961 \\
\hline 0.6 & $2.768075 \times 10^{-8}$ & 0.000060 & 7.523945 \\
\hline 0.8 & $3.677249 \times 10^{-7}$ & 0.000805 & 9.819431 \\
\hline 1 & $2.730839 \times 10^{-6}$ & 0.006037 & 11.986216 \\
\hline 1.2 & $1.403454 \times 10^{-5}$ & 0.031357 & 14.084697 \\
\hline 1.4 & $5.593443 \times 10^{-5}$ & 0.125637 & 16.279957 \\
\hline 1.6 & $1.850367 \times 10^{-4}$ & 0.425032 & 18.912513 \\
\hline 1.8 & $5.308537 \times 10^{-4}$ & 1.485475 & 22.603231 \\
\hline 2 & $1.360919 \times 10^{-3}$ & 6.557053 & 28.398776 \\
\hline
\end{tabular}

4.2 Example 2 Consider a nonlinear ordinary differential equation[28]

$y_{t t t}-t y_{t t}+t^{2} y^{2}=f(t)$

Subject the initial condition

$y(0)=0, y_{t}(0)=1, y_{t t}(0)=1$

The exact solution when $f(t)=t \sin (t)-\cos (t)+t^{2} \sin (t)^{2}$ is

$y(t)=\sin (t)$.

4.2.1. NHAM solution: To solve (4.13-4.14) by means of the NHAM, expanding the homotopy $\mu(x, t ; \mathbb{q})$ in powers of the parameter $\mathbb{q}$ with $s=3$ :

$\mu(t ; \mathbb{q})=\sum_{r=0}^{\infty} f_{r}^{3}(t) \mathbb{q}^{r}=f_{0}{ }^{3}(t) \mathbb{q}^{0}+f_{1}{ }^{3}(t) \mathbb{q}^{1}+\cdots+f_{n}{ }^{3}(t) \mathbb{q}^{n}+\cdots$,

where 
$f_{r}^{3}(t)=\frac{1}{3 r !}\left[\frac{d^{3 r}}{d t^{3 r}} f(t)\right]_{t=0} t^{3 r}+\frac{1}{(3 r+1) !}\left[\frac{d^{(3 r+1)}}{d t^{(3 r+1)}} f(t)\right]_{t=0} t^{(3 r+1)} \frac{1}{(3 r+2) !}\left[\frac{d^{(3 r+2)}}{d t^{(3 r+2)}} f(t)\right]_{t=0} t^{(3 r+2)}$

such that

$f_{0}^{3}(t)=-1+\frac{3 t^{2}}{2} \quad, f_{1}{ }^{3}(t)=\frac{19 t^{4}}{24} \quad, f_{2}{ }^{3}(t)=-\frac{233 t^{6}}{720}+\frac{1783 t^{8}}{40320} \quad, f_{3}{ }^{3}(t)=-\frac{11509 t^{10}}{3628800}, \ldots$

Let we choose the initial approximation

$y_{0}(t)=t$

And the linear operator

$$
L[\delta(t ; q)]=\frac{\partial^{3} \delta(t ; \mathbb{q})}{\partial t^{3}}
$$

with the property $L[c]=0$,

where $c$ is a constant of integration.

The nonlinear operator to the problem (4.13-4.14) under NHAM define as

$\mathbb{N}[\delta(t ; q)]=\frac{\partial^{3} \delta(t ; \mathbb{q})}{\partial t^{3}}-t \frac{\partial^{2} \delta(t ; q)}{\partial t^{2}}+t^{2} \delta^{2}(t ; q)-\mu(t ; q)$,

According to (2.4), the zero order deformation equation with the initial approximation (4.19) and linear operator (4.20) with (4.21) will be:

$(1-q) \mathrm{L}\left[\delta(t ; \mathfrak{q})-y_{0}(t)\right]=\mathbb{q} \mathbb{h} \mathbb{N}[\delta(t ; \mathfrak{q})-\mu(t ; \mathfrak{q})]$

and the $r$ th order deformation equation as follows:

$L\left[y_{r}(t)-X_{r} y_{r-1}(t)\right]=\mathbb{h} \mathcal{R}_{r}\left(\overrightarrow{y_{r-1}}(t)\right)$,

with the initial conditions $y_{r}(x, 0)=0, y_{r_{t}}(x, 0)=1$ and $y_{r_{t t}}(x, 0)=0$

Where

$\mathcal{R}_{r}\left(\overrightarrow{y_{r-1}}(t)\right)=y_{\mathrm{ttt}_{\mathrm{r}-1}}(t)-t y_{t t_{r-1}}(t)+t^{2} \sum_{i=0}^{r-1} y_{i} y_{r-1-i}-f_{r-1}^{3}(t)$

Now, the solution of (4.24)for $r \geq 1$ becomes

$y_{r}(t)=X_{r} y_{r-1}(t)+\mathbb{h} L^{-1} \mathcal{R}_{r}\left(\overrightarrow{y_{r-1}}(t)\right)$

And the following results are obtained:

$$
\begin{aligned}
& y_{1}(t)=\frac{1}{840} \mathfrak{h} t^{3}\left(140-21 t^{2}+4 t^{4}\right) \\
& y_{2}(t)=\frac{1}{840} \mathfrak{h} t^{3}\left(140-21 t^{2}+4 t^{4}\right)+\mathbb{h}\left(-\frac{19 t^{7}}{5040}+\mathbb{h}\left(\frac{t^{3}}{6}-\frac{t^{5}}{24}+\frac{t^{7}}{140}+\frac{t^{9}}{3780}-\frac{t^{11}}{19800}+\frac{t^{13}}{180180}\right)\right)
\end{aligned}
$$


Then, the series solution of the NHAM is:

$y(t, \mathbb{h}) \cong Y_{R}(t, \mathfrak{h})=\sum_{i=0}^{R} y_{i}(t, \mathbb{h})$

Equation (4.27) is an approximation solutions for the problem (4.13)-(4.14) depending on the parameters $\mathbb{h}$.

4.2.2. NMHAM solution: in order to solve (4.13) )-(4.14)by the proposed approach (NMHAM) we construct a system of differential equations as follows :

$y_{t}(t)=v(t)$

$v_{t}(t)=z(t)$

$z_{t}(t)=t z(t)-t^{2} y^{2}(t)+\mu(t ; \mathfrak{q})$

with the following initial conditions:

$y_{0}(t)=0$

$v_{0}(t)=1$

$z_{0}(t)=0$

Expanding the homotopy $\mu(t ; \mathfrak{q})$ in powers of the parameter $\mathbb{q}$ with $s=1$ :

$\mu(t ; \mathfrak{q})=\sum_{r=0}^{\infty} f_{r}{ }^{1}(t) \mathbb{q}^{r}=f_{0}{ }^{1}(t) \mathbb{q}^{0}+f_{1}{ }^{1}(t) \mathbb{q}^{1}+\cdots+f_{n}{ }^{1}(t) \mathbb{q}^{n}+\cdots$,

Where

$f_{r}^{1}(t)=\frac{1}{r !}\left[\frac{d^{r}}{d t^{r}} f(t)\right]_{t=0} t^{r}$

such that

$f_{0}^{1}(t)=-1, \quad f_{1}^{1}(t)=0, \quad f_{2}^{1}(t)=\frac{3 t^{2}}{2}, \cdots$

And the following linear operators:

$L y(t)=\frac{\partial y(t)}{\partial t}, L v(t)=\frac{\partial v(t)}{\partial t}, L z(t)=\frac{\partial z(t)}{\partial t}$

$A y_{r-1}(t)=-f_{r-1}^{1}(t)$ 
$B y_{r-1}(x, t)=-t z_{r-1}(t)+t^{2} \sum_{i=0}^{r-1} y_{i} y_{r-1-i}$

We obtain

$y_{1}(t)=\mathbb{n} \int_{0}^{t}\left(-v_{0}(\tau)\right) d \tau$

$v_{1}(t)=\mathbb{h} \int_{0}^{t}\left(-z_{0}(\tau)\right) d \tau$

$z_{1}(t)=\mathbb{h} \int_{0}^{t}\left(-t z_{0}(\tau)+t^{2} y_{0}^{2}(\tau)-f_{0}^{1}(\tau)\right) d \tau$

Now, for $r \geq 2$, we get

$y_{r}(t)=(1+\mathbb{h}) y_{r-1}(t)+\mathbb{h} \int_{0}^{t}\left(-v_{r-1}(\tau)\right) d \tau$

$v_{r}(t)=(1+\mathbb{h}) v_{r-1}(t)+\mathbb{h} \int_{0}^{t}\left(-z_{r-1}(\tau)\right) d \tau$

$z_{r}(t)=(1+\mathbb{h}) z_{r-1}(t)+\mathbb{h} \int_{0}^{t}\left(A y_{r-1}(\tau)+B\left(y_{r-1}(\tau)\right)\right) d \tau$

And the following results are obtained:

$y_{1}(t)=-\mathbb{h} t, v_{1}(t)=0, z_{1}(t)=\mathbb{h} t$

$y_{2}(t)=-\mathbb{h}(1+\mathbb{h}) t, v_{2}(t)=-\frac{1}{2} \mathbb{h}^{2} t^{2}, z_{2}(t)=\mathbb{h}(1+\mathbb{h}) t-\frac{\mathbb{h}^{2} t^{3}}{3}$,

$y_{3}(t)=-\mathbb{h}(1+\mathbb{n})^{2} t+\frac{\mathbb{h}^{3} t^{3}}{6}$,

$\vdots$

Then, the series solution of the NMHAM is:

$y(t, \mathbb{l}) \cong Y_{R}(t, \mathbb{h})=\sum_{i=0}^{R} y_{i}(t, \mathbb{h})$

Equation (4.37) is an approximation solutions for the problem (4.13)-(4.14) depending on the parameter $\mathbb{h}$. To determine the valid region of $\mathbb{h}$, the $\mathbb{h}$-curves given by the $7^{\text {th }}$ order of NMHAM at different values of $t$ are drawn in figure (8). 


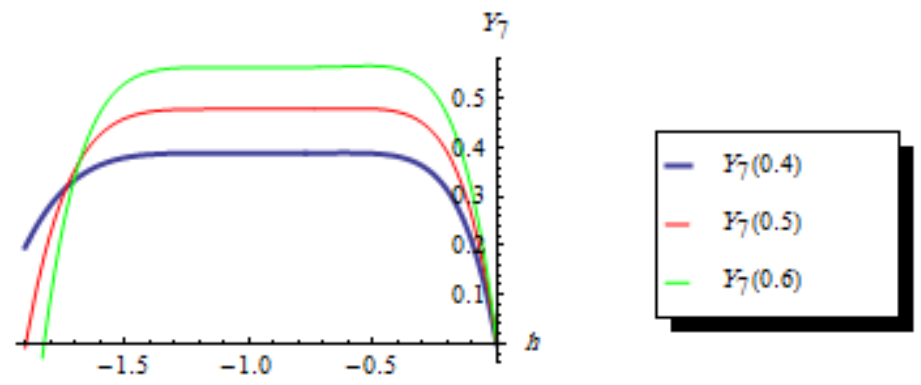

Figure(8): $\mathbb{l}$-curve for NMHAM approximation solutions $\mathrm{Y}_{7}$ of problem (4.13)-(4.14) at different values of $t$.

Figure(9) shows the comparison between $Y_{7}$ of NMHAM and $Y_{7}$ of NHAM at $0 \leq t \leq 2$, with the exact solution(4.15) which indicates that the series solution $Y_{7}$ obtained by MNHAMis more accurate from the series solution $Y_{7}$ obtained by NHAM.Figure (10) shows the comparison of $Y_{6}$, $Y_{5}$ of NMHAM and $Y_{7}$ of NHAM with the exact solution (4.15) at $0 \leq t \leq 1.5$ which indicates that the speed of convergence of NMHAM is faster and more convergence in comparison of NHAM.Figures (11) shows that the series solution $Y_{7}$ obtained by NMHAM at $0 \leq t \leq 1$ is more accurate from the series solution $Y_{7}$ obtained by NHAMFigures (12) shows that the series solution $Y_{6}$ obtained by NMHAM is more and faster convergence from the series solution $Y_{7}$ obtained by NHAM at $0 \leq t \leq 1$.
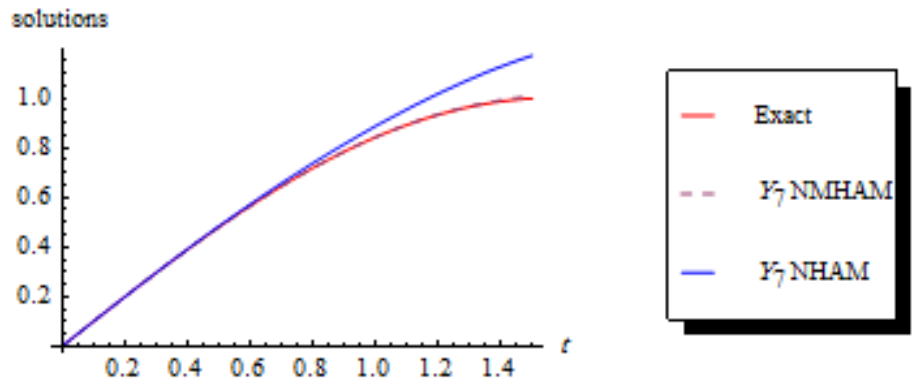

Figure(9): Comparison of the $7^{\text {th }}$ order approximations of NMHAM and NHAM at $0 \leq t \leq 1.5$, $\mathbb{h}=-1$ with the exact solution(4.15).
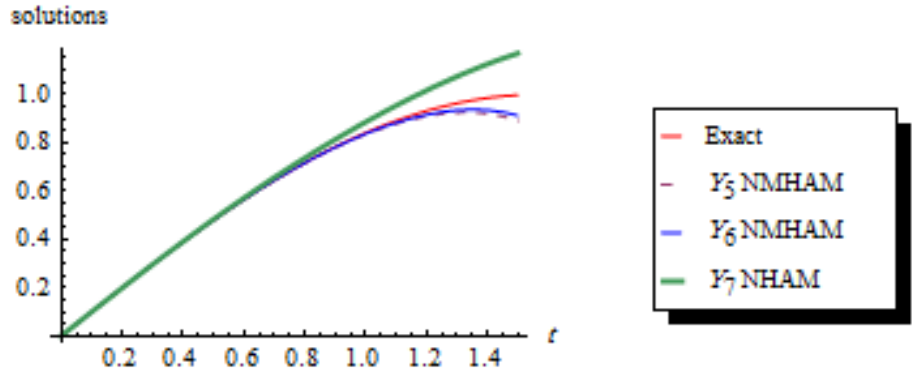

Figure(10):Comparison of $Y_{6}$ and $Y_{5}$ of NMHAM and $Y_{7}$ of NHAM with the exact solution (4.15) at $0 \leq t \leq 1.5 \mathbb{h}=-1$. 

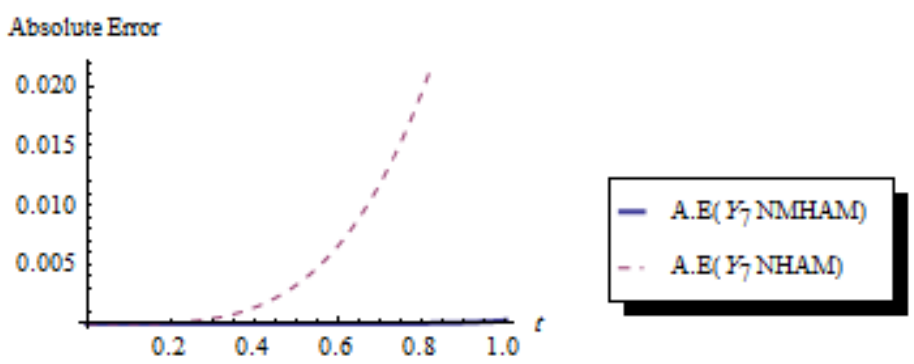

Figure(11): The absolute errors of the $7^{\text {th }}$ order approximations of NMHAM and NHAM at $0 \leq t \leq 1$, $\mathbb{h}=-1$.
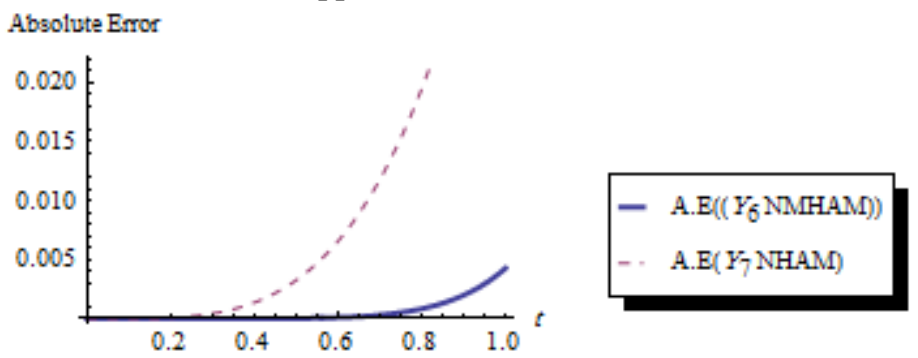

Figure(12):The absolute errors of the $6^{\text {th }}$ order approximations of NMHAM and the $7^{\text {th }}$ order approximations of NHAM at $0 \leq t \leq 1, \mathbb{h}=-1$.

Table (3) show the comparison of $Y_{7}$ of NMHAM ,NHAM and nHAM ,with the exact solution (4.15) at different values of of $t$.Table (4) shows the comparison of the absolute errors of $Y_{7}$ of NMHAM ,NHAM and nHAM at different values of of $t$. Tables (3) and (4) indicate that the series solution obtained by NMHAM is more accurate from the series solution obtained by NHAM and the series solution obtained by nHAM is divergent.

Table3: Comparison of the $7^{\text {th }}$ order approximations of NMHAM, NHAM and nHAM with the exact solution (4.15).

\begin{tabular}{|c|c|c|c|c|}
\hline $\boldsymbol{t}$ & Exact & NMHAM & NHAM & nHAM \\
\hline 0 & 0 & 0 & 0 & 0 \\
\hline 0.1 & 0.099833 & 0.099833 & 0.099839 & -350.966 \\
\hline 0.2 & 0.198669 & 0.198669 & 0.198762 & -701.933 \\
\hline 0.3 & 0.295520 & 0.295520 & 0.295974 & -1052.902 \\
\hline 0.4 & 0.389418 & 0.389418 & 0.390805 & -1403.874 \\
\hline 0.5 & 0.479426 & 0.479426 & 0.482704 & -1754.851 \\
\hline 0.6 & 0.564642 & 0.564646 & 0.571229 & -2105.831 \\
\hline 0.7 & 0.644218 & 0.644230 & 0.656040 & -2456.816 \\
\hline 0.8 & 0.717356 & 0.717397 & 0.736880 & -2807.808 \\
\hline 0.9 & 0.783327 & 0.783442 & 0.813554 & -3158.805 \\
\hline 1 & 0.841471 & 0.841760 & 0.885889 & -3509.807 \\
\hline
\end{tabular}


Table4: Comparison of the absolute errors of $7^{\text {th }}$ order approximations of NMHAM , NHAM and nHAM with the exact solution(4.15).

\begin{tabular}{|c|c|c|c|}
\hline $\boldsymbol{t}$ & $\boldsymbol{A . E} \boldsymbol{N M H A M}$ & $\boldsymbol{A . E} \boldsymbol{N H A M}$ & $\boldsymbol{A . E} \boldsymbol{n H A M}$ \\
\hline 0 & 0 & 0 & 0 \\
\hline 0.1 & $3.275435 \times 10^{-13}$ & $6.012234 \times 10^{-6}$ & 351.066 \\
\hline 0.2 & $1.671137 \times 10^{-10}$ & $9.274402 \times 10^{-5}$ & 702.132 \\
\hline 0.3 & $6.386566 \times 10^{-9}$ & $4.535345 \times 10^{-4}$ & 1053.198 \\
\hline 0.4 & $8.435251 \times 10^{-8}$ & 0.0013867 & 1053.198 \\
\hline 0.5 & $6.217155 \times 10^{-7}$ & 0.003279 & 1755.329 \\
\hline 0.6 & $3.165286 \times 10^{-6}$ & 0.006587 & 2106.396 \\
\hline 0.7 & $1.247286 \times 10^{-5}$ & 0.011823 & 2457.461 \\
\hline 0.8 & $4.071103 \times 10^{-5}$ & 0.019524 & 2808.526 \\
\hline 0.9 & $1.149799 \times 10^{-4}$ & 0.030227 & 3159.588 \\
\hline 1 & $2.894769 \times 10^{-4}$ & 0.044418 & 3510.648 \\
\hline
\end{tabular}

4.3 Example 3 Consider non-linear Klein-Gordon equation as follows [1]

$y_{t t}-y_{x x}+y^{2}=f(x, t)$

Subject the initial condition

$y(x, 0)=0, y_{t}(x, 0)=0$

The exact solution when $f(x, t)=2 x^{2}-2 t^{2}+x^{4} t^{4}$ is

$y(x, t)=x^{2} t^{2}$

4.3.1. NHAM solution: To solve (4.38-4.39) by means of the NHAM, expanding the homotopy $\mu(x, t ; \mathbb{q})$ in powers of the parameter $\mathbb{q}$ with $s=2$ :

$$
\mu(x, t ; q)=\sum_{r=0}^{\infty} f_{r}^{2}(x, t) \mathbb{q}^{r}=f_{0}^{2}(x, t) \mathbb{q}^{0}+f_{1}^{2}(x, t) \mathbb{q}^{1}+\cdots+f_{n}^{2}(x, t) \mathbb{q}^{n}+\cdots
$$

Where

$f_{r}^{2}(x, t)=\frac{1}{2 r !}\left[\frac{d^{2 r}}{d t^{2 r}} f(x, t)\right]_{t=0} t^{2 r}+\frac{1}{(2 r+1) !}\left[\frac{d^{(2 r+1)}}{d t^{(2 r+1)}} f(x, t)\right]_{t=0} t^{(2 r+1)}$

such that

$f_{0}^{2}(x, t)=2 x^{2} \quad, f_{1}^{2}(x, t)=-2 t^{2} \quad, f_{2}^{2}(x, t)=t^{4} x^{4} \quad, f_{3}^{2}(x, t)=0, \ldots$

Let we choose the initial approximation

$y_{0}(x, t)=0$,

And the linear operator 
$L[\delta(x, t ; \mathbb{q})]=\frac{\partial^{2} \delta(x, t ; \mathbb{q})}{\partial t^{2}}$

with the property $L[c]=0$,

where $c$ is a constant of integration.

The problem (4.38-4.39) under NHAM suggests to define a nonlinear operator as

$\mathbb{N}[\delta(x, t ; \mathbb{q})]=\frac{\partial^{2} \delta(x, t ; q)}{\partial t^{2}}-\frac{\partial^{2} \delta(x, t ; q)}{\partial x^{2}}+\delta^{2}(x, t ; \mathbb{q})-\mu(x, t ; \mathbb{q})$,

According to (2.4), the zero order deformation equation with the initial approximation (4.44) and linear operator (4.45) with (4.46) will be:

$(1-\mathbb{q}) \mathrm{L}\left[\delta(x, t ; \mathfrak{q})-y_{0}(x, t)\right]=\mathbb{q} \mathbb{L} \mathbb{N}[\delta(x, t ; \mathfrak{q})-\mu(x, t ; \mathbb{q})]$,

and the $r$ th order deformation equation as follows:

$L\left[y_{r}(x, t)-X_{r} y_{r-1}(x, t)\right]=\mathbb{h} \mathcal{R}_{r}\left(\overrightarrow{y_{r-1}}(x, t)\right)$,

with the initial conditions $y_{r}(x, 0)=0$ and $y_{r_{t}}(x, 0)=0$

Where

$\mathcal{R}_{r}\left(\overrightarrow{y_{r-1}}(x, t)\right)=y_{\mathrm{tt}_{\mathrm{r}-1}}(x, t)-y_{x x_{r-1}}(x, t)+\sum_{i=0}^{r-1} y_{i} y_{r-1-i}-f^{2}{ }_{r-1}(x, t)$

Now, the solution of (4.49)for $r \geq 1$ becomes

$y_{r}(x, t)=X_{r} y_{r-1}(x, t)+\mathbb{n} L^{-1} \mathcal{R}_{r}\left(\overrightarrow{y_{r-1}}(x, t)\right)$

We now successively obtain

$y_{1}(x, t)=-\llbracket n t^{2} x^{2}$

$y_{2}(x, t)=-\mathbb{h} t^{2} x^{2}+\frac{1}{6} \mathbb{h}\left((1+\mathbb{h}) t^{4}-6 \mathfrak{h} t^{2} x^{2}\right.$

:

Then, the series solution of the NHAM is:

$y(x, t, \mathbb{h}) \cong Y_{R}(x, t, \mathbb{h})=\sum_{i=0}^{R} y_{i}(x, t, \mathbb{h})$

Equation (4.52) is a family of approximation solutions to the problem (4.38)-(4.39) in terms of the convergence parameters $\mathbb{h}$.

4.3.2. NMHAM solution: in order to solve (4.38) )-(4.39)by the proposed approach (NMHAM) we construct the following system:

$y_{t}(x, t)=v(x, t)$,

$v_{t}(x, t)=y_{x x}-y^{2}+\mu(x, t ; \mathbb{q})$

with the following initial conditions 
$y_{0}(x, t)=0, v_{0}(x, t)=0$

Expanding the homotopy $\mu(x, t ; \mathbb{q})$ in powers of the parameter $\mathbb{q}$ with $s=1$ :

$\mu(x, t ; \mathfrak{q})=\sum_{r=0}^{\infty} f_{r}{ }^{1}(x, t) \mathbb{q}^{r}=f_{0}{ }^{1}(x, t) \mathbb{q}^{0}+f_{1}{ }^{1}(x, t) \mathbb{q}^{1}+\cdots+f_{n}{ }^{1}(x, t) \mathbb{q}^{n}+\cdots$,

Where

$f_{r}^{1}(x, t)=\frac{1}{r !}\left[\frac{d^{r}}{d t^{r}} f(x, t)\right]_{t=0} t^{r}$

such that

$f_{0}{ }^{1}(x, t)=\frac{5}{4} \quad, f_{1}{ }^{1}(x, t)=0 \quad, f_{2}{ }^{1}(x, t)=\frac{t^{2}}{16} \quad, f_{3}{ }^{1}(x, t)=0, \ldots$

and the following linear operators:

$L y(x, t)=\frac{\partial y(x, t)}{\partial t}, L v(x, t)=\frac{\partial v(x, t)}{\partial t}$

$A y_{r-1}(t)=-y_{x x_{r-1}}(x, t)-f_{r-1}^{1}(x, t)$

$B y_{r-1}(x, t)=\sum_{i=0}^{r-1} y_{i} y_{r-1-i}$

we obtain

$y_{1}(x, t)=\mathbb{h} \int_{0}^{t}\left(-v_{0}(x, \tau)\right) d \tau$

$v_{1}(x, t)=\mathbb{h} \int_{0}^{t}\left(-y_{0_{x x}}(x, \tau)+y_{0}{ }^{2}(x, \tau)-f_{0}{ }^{1}(x, \tau)\right) d \tau$

Now, for $r \geq 2$, we get

$y_{r}(x, t)=(1+\mathbb{h}) y_{r-1}(x, t)+\mathbb{h} \int_{0}^{t}\left(-v_{r-1}(x, \tau)\right) d \tau$,

$v_{r}(x, t)=(1+\mathbb{h}) v_{r-1}(x, t)+\mathbb{h} \int_{0}^{t}\left(A y_{r-1}(x, \tau)+B y_{r-1}(x, \tau)\right) d \tau$

And the following results are obtained:

$y_{1}(x, t)=0, \quad v_{1}(x, t)=-2 \rrbracket t x^{2}$

$y_{2}(x, t)=\mathbb{h}^{2} t^{2} x^{2}, \quad v_{2}(x, t)=-2 \mathbb{h}(1+\mathbb{h}) t x^{2}$

$y_{3}(x, t)=\mathbb{h}^{2}(1+\mathbb{h}) t^{2} x^{2}+\mathbb{h}\left(\mathbb{h} t^{2} x^{2}+\mathbb{h}^{2} t^{2} x^{2}\right)$

$\vdots$

Then, the series solution of the NMHAM is: 
$y(x, t, \mathbb{h}) \cong Y_{R}(x, t, \mathbb{h})=\sum_{i=0}^{R} y_{i}(x, t, \mathbb{h})$

Equation (4.61) is an approximation solutions for the problem (4.38)- (4.39) depending on the parameter $\mathbb{l}$.To determine the valid region of $\mathbb{h}$, the $\mathbb{h}$-curves given by the $5^{\text {th }}$-order NMHAM at different values of $x$ and $t$ are drawn in figure (13).
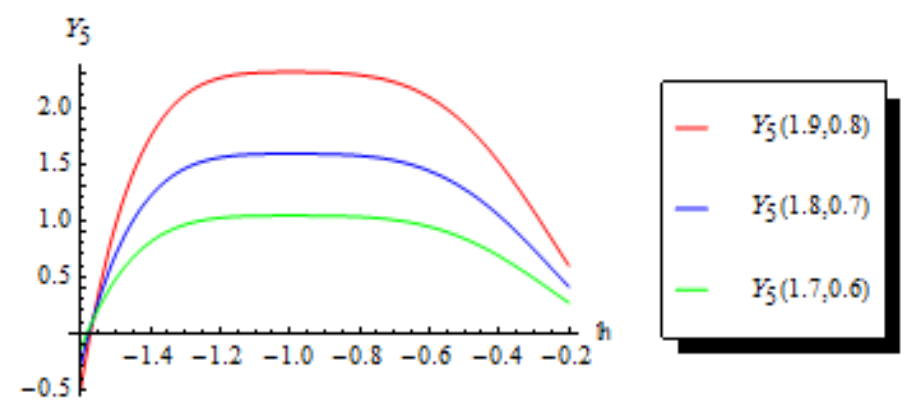

Figure (13): $\mathbb{h}$-curve for NMHAM approximation solutions $Y_{5}$ of problem (4.38)-(4.39) at different values of $x$ and $t$.

Figures (14) show that the series solution $Y_{5}$ obtained by NMHAM at $0 \leq t \leq 1.5, x=0.2$ is more accurate from the series solution $Y_{5}$ obtained by NHAM.Figures (15) show that the series solution $Y_{5}$ obtained by NMHAM is more accurate from the series solution $Y_{5}$ obtained by NHAM at larger $t(1.5 \leq t \leq 3, x=0.2)$.

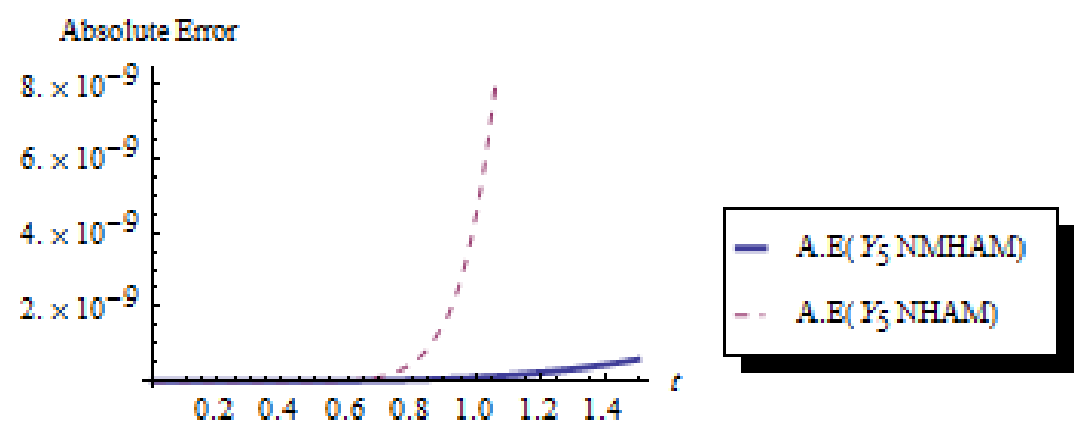

Figure(14): The absolute errors of $Y_{5}$ of NMHAM and NHAM at $0 \leq t \leq 1.5, x=0.2$, $\mathrm{h}=-0.99$.

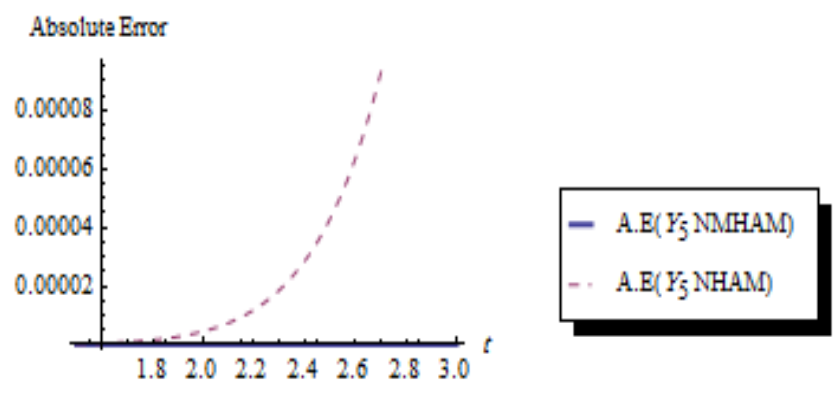

Figure(15):The absolute errors of $Y_{5}$ of NMHAM and NHAM at $1.5 \leq t \leq 3, x=0.2$, $\mathbb{h}=-0.99$. 
Table (5) show the comparison of $Y_{5}$ of NMHAM ,NHAM and nHAM ,with the exact solution (4.40). Table (6) shows the comparison between the absolute errors of $Y_{5}$ of NMHAM ,NHAM and nHAM. Tables (5) and (6) indicate that the series solution obtained by NMHAM is more accurate from the series solution obtained by NHAM and nHAM.

Table5: Comparison of the $5^{\text {th }}$ order approximations of NMHAM , NHAM and nHAM at different values of $t$ and $x$ with the exact solution(4.40).

\begin{tabular}{|c|c|c|c|c|c|}
\hline$x$ & $t$ & exact & NMHAM & NHAM & nHAM \\
\hline \multirow[t]{6}{*}{0.2} & 0 & 0 & 0 & 0 & 0 \\
\hline & 0.5 & 0.01 & 0.009999 & 0.010000 & 0.010002 \\
\hline & 1 & 0.04 & 0.039999 & 0.040000 & 0.040339 \\
\hline & 1.5 & 0.09 & 0.089999 & 0.090000 & 0.097930 \\
\hline & 2 & 0.16 & 0.159999 & 0.160000 & 0.236556 \\
\hline & 2.5 & 0.25 & 0.249999 & 0.250004 & 0.698986 \\
\hline \multirow[t]{6}{*}{0.6} & 0 & 0 & 0 & 0 & 0 \\
\hline & 0.5 & 0.09 & 0.089999 & 0.090000 & 0.090078 \\
\hline & 1 & 0.36 & 0.359999 & 0.360000 & 0.366891 \\
\hline & 1.5 & 0.809999 & 0.809999 & 0.8100000 & 0.925110 \\
\hline & 2 & 1.44 & 1.439999 & 1.440000 & 2.374766 \\
\hline & 2.5 & 2.25 & 2.249999 & 2.250004 & 7.228376 \\
\hline \multirow[t]{6}{*}{1} & 0 & 0 & 0 & 0 & 0 \\
\hline & 0.5 & 0.25 & 0.249999 & 0.249999 & 0.250549 \\
\hline & 1 & 1 & 0.999999 & 0.999999 & 1.040476 \\
\hline & 1.5 & 2.25 & 2.249999 & 2.249999 & 2.812751 \\
\hline & 2 & 4 & 3.999999 & 3.999998 & 7.961905 \\
\hline & 2.5 & 6.25 & 6.249999 & 6.249990 & 25.287156 \\
\hline
\end{tabular}

Table 6:Comparison of the absolute errors of $5^{\text {th }}$ order approximations of NMHAM , NHAM and nHAM with the exact solution (4.40).

\begin{tabular}{|c|c|c|c|c|}
\hline $\boldsymbol{x}$ & $\boldsymbol{t}$ & $\boldsymbol{A . E}$ NMHAM & A.E NHAM & A.E $\boldsymbol{n H A M}$ \\
\hline 0.2 & 0 & 0 & 0 & 0 \\
\cline { 2 - 5 } & 0.5 & $7.291737 \times 10^{-14}$ & $4.391019 \times 10^{-13}$ & $1.949405 \times 10^{-6}$ \\
\cline { 2 - 5 } & 1 & $1.166678 \times 10^{-12}$ & $4.496417 \times 10^{-10}$ & $3.390476 \times 10^{-4}$ \\
\cline { 2 - 5 } & 1.5 & $5.906234 \times 10^{-12}$ & $2.592851 \times 10^{-8}$ & 0.007930 \\
\cline { 2 - 5 } & 2 & $1.866693 \times 10^{-11}$ & $4.604308 \times 10^{-7}$ & 0.076556 \\
\cline { 2 - 5 } & 2.5 & $4.557352 \times 10^{-11}$ & $4.288093 \times 10^{-6}$ & 0.448986 \\
\hline 0.6 & 0 & 0 & 0 & 0 \\
\cline { 2 - 5 } & 0.5 & $7.294165 \times 10^{-14}$ & $3.717859 \times 10^{-13}$ & $7.754464 \times 10^{-5}$ \\
\cline { 2 - 5 } & 1 & $1.166733 \times 10^{-12}$ & $3.806401 \times 10^{-10}$ & 0.006891 \\
\cline { 2 - 5 } & 1.5 & $5.906164 \times 10^{-12}$ & $2.194879 \times 10^{-8}$ & 0.115110 \\
\cline { 2 - 5 } & 2 & $1.866729 \times 10^{-11}$ & $3.897552 \times 10^{-7}$ & 0.934766 \\
\cline { 2 - 5 } 1 & 2.5 & $4.557421 \times 10^{-11}$ & $3.629856 \times 10^{-6}$ & 4.978376 \\
\cline { 2 - 5 } & 0 & 0 & 0 & 0 \\
\cline { 2 - 5 } & 0.5 & $7.291389 \times 10^{-14}$ & $1.007444 \times 10^{-12}$ & $5.487351 \times 10^{-4}$ \\
\cline { 2 - 5 } & 1 & $1.166622 \times 10^{-12}$ & $1.031669 \times 10^{-9}$ & 0.040476 \\
\hline
\end{tabular}




\begin{tabular}{|c|c|c|c|c|}
\hline \multirow{2}{*}{1.5} & $5.907275 \times 10^{-12}$ & $5.949359 \times 10^{-8}$ & 0.562751 \\
\cline { 2 - 5 } & 2 & $1.866685 \times 10^{-11}$ & $1.056486 \times 10^{-6}$ & 3.961905 \\
\cline { 2 - 5 } & 2.5 & $4.557509 \times 10^{-11}$ & $9.839353 \times 10^{-6}$ & 19.037156 \\
\hline
\end{tabular}

\section{Conclusion}

In this article, new powerful modification of homotopy analysis method (NMHAM) was proposed to create an approximate solution of nonhomogeneous nonlinear ordinary and partial differential equations. The main advantage of the NMHAM is that it requires less computational work compared with the NHAM and nHAM in finding approximate solutions for nonlinear nonhomogeneous differential equations. Illustrative examples show that the series solution obtained by NMHAM is more accurate from the series solution obtained byNHAM andnHAM. Therefore, depending on the results of this work, we can say that the NMHAM is more effective than NHAM and nHAM.

\section{References}

[1] Ayati Z., Biazar J., Gharedaghi B.,"The application of modified homotopy analysis method for solving linear and non-linear inhomogeneous Klein-Gordon equations"Acta Universitatis Apulensis, no. 39,pp. 31-40,2014.

[2] Bataineh, A.S., M.S.M. Noorani and I. Hashim, 2008. Phys. Lett., A 372: 4062.

[3] Bataineh, A.S., M.S.M. Noorani and I. Hashim, 2009. Commun. Nonlinear Sci. Numer. Simulat., 14: 409.

[4] Bataineh, A.S., Avoid the uncontrollability problems of the nonzero endpoint conditions via homotopy analysis method, Appl. Comput. Math., V.13, N.1, 2014, pp. 1-13

[5] El-TawilM. A. and HuseenS.N., The q-Homotopy Analysis Method (q-HAM), International Journal of Applied mathematics and mechanics, 8 (15): 51-75, 2012.

[6] El-TawilM. A. and HuseenS.N., On Convergence of The q-Homotopy Analysis Method, Int. J. Contemp. Math. Sciences, Vol. 8, 2013, no. 10, $481-497$.

[7] Hassan H.N. and El-Tawil M.A., "A new technique of using homotopy analysis method for solving high-order nonlinear differential equations," Mathematical Method in the Applied Sciences, vol.34,no.6,pp.728-742,2011.

[8] Huseen S. N. and Grace, S. R. 2013, Approximate Solutions of Nonlinear Partial Differential Equations by Modified q-Homotopy Analysis Method (mq-HAM), HindawiPublishing Corporation, Journal of Applied Mathematics, Article ID 569674, 9 pages http:// dx.doi.org/10.1155/2013/ 569674.

[9] Huseen S. N., Grace S. R. and El-Tawil M. A. 2013, The Optimal q-Homotopy Analysis Method (Oq-HAM), International Journal of Computers \& Technology, Vol 11, No. 8.

[10] Huseen S. N., Solving the K(2,2) Equation by Means of the q-Homotopy Analysis Method (q-HAM), International Journal of Innovative Science, Engineering \& Technology, Vol. 2 Issue 8, August 2015.

[11] Huseen S. N., Series Solutions of Fractional Initial-Value Problems by q-Homotopy Analysis Method, International Journal of Innovative Science, Engineering \& Technology, Vol. 3 Issue 1, January 2016.

[12] Huseen Shaheed N. Application of optimal q-homotopy analysis method to second order initial and boundary value problems. Int $\mathrm{J}$ Sci Innovative Math Res (IJSIMR) 2015;3(1):18-24. 
[13] Huseen S. N., A Numerical Study of One-Dimensional HyperbolicTelegraph Equation, Journal of Mathematics and System Science 7 (2017) 62-72.

[14] Liao S. J., Proposed homotopy analysis techniques for the solution of nonlinear problems, Ph.D. dissertation, Shanghai Jiao Tong University, Shanghai, 1992.

[15] Liao S. J., An approximate solution technique which does not depend upon smallparameters: a special example, Int. J. Non-linear Mech. 30:371-380(1995).

[16] Liao S. J., An approximate solution technique which does not depend upon small parameters (Part 2): an application in fluid mechanics, Int. J. Non-linear Mech. 32:815822(1997).

[17] Liao S. J., An explicit, totally analytic approximation of Blasius viscous flow problem, Int. J.Non-Linear Mech. 34:759-778(1999).

[18] Liao S. J., A uniformly valid analytic solution of 2D viscous flow past a semi-infinite flat plate. J. Fluid Mech. 385:101-128(1999).

[19] Liao S. J., A. Campo, Analytic solutions of the temperature distribution in Blasius viscous flow problems, J.Fluid Mech. 453:411-425(2002).

[20] Liao S. J., An explicit analytic solution to the Thomas-Fermi equation, Appl. Math.Comput. 144: 495-506(2003).

[21] Liao S. J., On the analytic solution of magnetohydrodynamic flows of non-Newtonian fluids over a stretching sheet, J. Fluid Mech. 488:189-212(2003)

[22] Liao S. J., On the homotopy analysis method for nonlinear problems, Appl. Math. Comput.147: 499-513(2004).

[23] Liao S. J. and Magyari E., Exponentially decaying boundary layers as limiting cases of families of algebraically decaying ones, Z. Angew. Math. Phys. 57:777-792(2006).

[24] Liao S. J. and Tan Y., A general approach to obtain series solutions of nonlinear differential equations, Stud. Appl. Math. 119:297-354(2007).

[25] Liao S. J., A general approach to get series solution of non-similarity boundary-layer flows, Commun. Nonlinear Sci. Numer. Simulat. 14:2144-2159(2009).

[26] Liao S. J., Beyond perturbation: Introduction to the homotopy analysis method, CRC pressLLC, Boca Raton, 2003.

[27] Mahmoudi Y. and Kazemian M., Some Notes on Homotopy Analysis Method for Solving the Fornberg-Whitham Equation, J. Basic. Appl. Sci. Res., 2(3)2985-2990, 2012.

[28] Pue-on P. and Viriyapong V., Modified Adomian Decomposition Method for Solving Particular Third-Order ordinary Differential Equations . Appl. Math .Sci. ,Vol.6,2012, no.30,1436-1469. 